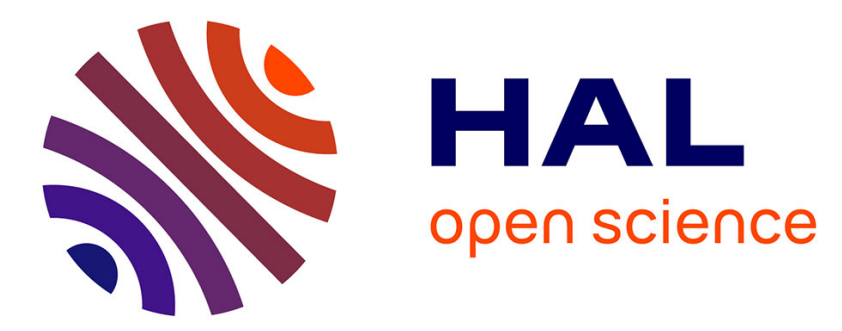

\title{
Les formations végétales méditerranéennes du versant nord cantabrique (Espagne du Nord-Ouest) : un problème écologique
}

Georges Bertrand

\section{- To cite this version: \\ Georges Bertrand. Les formations végétales méditerranéennes du versant nord cantabrique (Espagne du Nord-Ouest): un problème écologique. Bulletin de l'Association de géographes français, 1964, 328-329, pp.42-56. 10.3406/bagf.1964.5706 . hal-02570325}

\section{HAL Id: hal-02570325 \\ https://hal.science/hal-02570325}

Submitted on 11 May 2020

HAL is a multi-disciplinary open access archive for the deposit and dissemination of scientific research documents, whether they are published or not. The documents may come from teaching and research institutions in France or abroad, or from public or private research centers.
L'archive ouverte pluridisciplinaire HAL, est destinée au dépôt et à la diffusion de documents scientifiques de niveau recherche, publiés ou non, émanant des établissements d'enseignement et de recherche français ou étrangers, des laboratoires publics ou privés. 


\section{Les formations végétales méditerranéennes du versant nord} cantabrique (Espagne du Nord-Ouest) : un problème écologique Georges Bertrand

\section{Citer ce document / Cite this document :}

Bertrand Georges. Les formations végétales méditerranéennes du versant nord cantabrique (Espagne du Nord-Ouest) : un problème écologique. In: Bulletin de l'Association de géographes français, N³28-329, 41e année, Novembre-décembre 1964. pp. 42-56;

doi : https://doi.org/10.3406/bagf.1964.5706

https://www.persee.fr/doc/bagf_0004-5322_1964_num_41_328_5706

Fichier pdf généré le 25/04/2018 


\section{Communication de M. Georges Bertrand}

\section{Les formations végétales méditerranéennes du versant nord Cantabrique (Espagne du Nord-Ouest) : un problème écologique}

En pleine zone océanique, sur les falaises de la côte asturienne comme dans les bassins intra-montagnards des Picos de Europa, s'étendent des paysages végétaux que l'on associe, en général, au climat méditerranéen. Le Chêne Vert, le Chêne-Liège, le Pistachier, le Lentisque, le Genévrier Oxycèdre, le Jasmin jaune, la Lavande stoechas, etc., voisinent avec les espèces atlantiques et montagnardes des «séries » des Chênes à feuilles caduques et du Hêtre (1). Il s'agit moins de plantes isolées que de véritables «formations végétales» dont la structure et la dynamique interne paraissent identiques à celles de certains paysages végétaux de la région méditerranéenne. Pourtant, les conditions bioclimatiques d'ensemble ne paraissent guère comparables. Le versant nord cantabrique subit un climat hyperocéanique : tranche d'eau annuelle de 1.200 à $2.000 \mathrm{~mm}$ assez bien répartie dans l'année, nébulosité et degré hygrométrique toujours élevés, températures modérées en toutes saisons. La première impression est donc celle d'une dysharmonie entre la végétation et le milieu.

La vallée du Deva (fig. 1), qui se situe à la limite de la « Montaña » de Santander et de la province d'Asturias, possède une gamme variée de stations méditerranéennes. Elle se prête particulièrement bien à une étude écologique. Le Deva prend sa source en Liébana, à $1.100 \mathrm{~m}$ d'altitude. Dans son cours moyen et inférieur, le fleuve tranche perpendiculairement du Sud au Nord, une partie des structures hercyniennes et alpines du Massif Cantabrique avant de rejoindre 1"Océan dans la ria en «goulot de bouteille » de Tina Mayor (fig. 2). Cette disposition de la structure et des affleurements rocheux, en faisant alterner gorges et bassins, entraîne de rapides variations de la pente, des sols, des microclimats et des types de mise en valeur économique, éléments favorables à la diversité des biotopes. Du point de vue biogéographique, comme d'ail-

\footnotetext{
(1) Pour ne pas alourdir le texte on a utilisé, chaque fois que c'étalt possible,
} le nom des plantes en francais. 


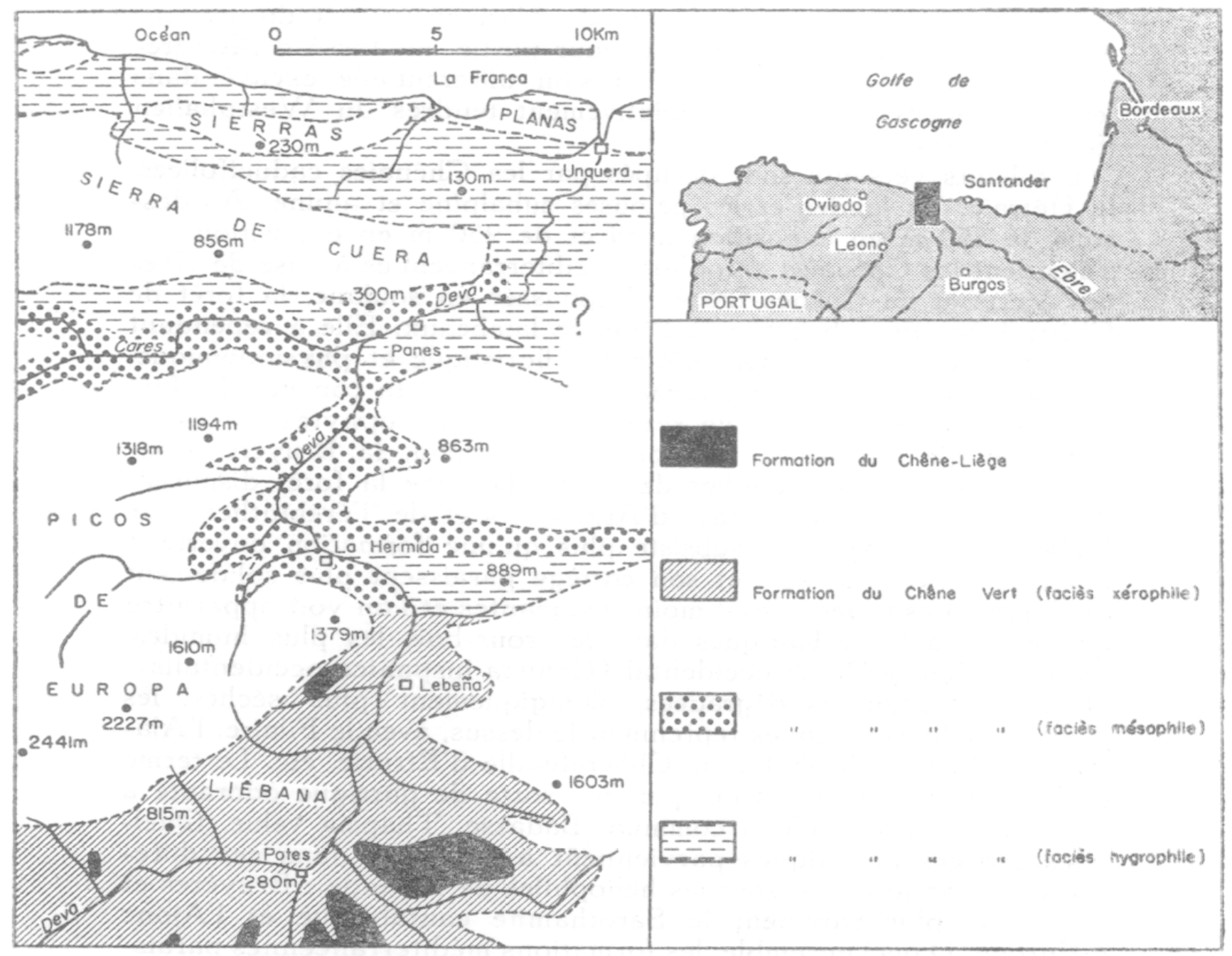

Figure 1 : Croquis de localisation et vallée du Deva : la dégradation écologique à l'intérieur des domaines biogéographiques méditerranéens (a la place de * formation * lire * domaine $\$$ ).

leurs du point de vue géomorphologique, il faut opposer la moyenne vallée abritée par le Massif des Picos de Europa à la basse vallée largement ouverte aux influences océaniques.

\section{LES STATIONS INTRA-MONTAGNARDES ABRITEES}

\section{A) Une enclave méditerranéenne : la basse Liébana}

La Liébana correspond au bassin supérieur du Deva. C'est une cuvette ovoïde de 20 à $25 \mathrm{~km}$ de diamètre, très déprimée en son centre ( $280 \mathrm{~m}$ à Potes) et cernée de tous côtés par des massifs de 2.000 à $2.600 \mathrm{~m}$ d'altitude taillés dans les calcaires cristallins 
du Dinantien et les conglomérats du Carbonifère ou du PermoTrias. La Liébana elle-même est, pour partie, un fossé d'effondrement, pour partie, un bassin d'érosion différentielle excavé dans les grauwackes et le flysch relativement tendres du Westphalien (faciès Culm).

Les hauts versants sont occupés par les Chênaies caducifoliées, la Hêtraie, les landes et les pelouses subalpine et alpine. Au-dessous de $850 \mathrm{~m}$ en exposition nord et de $950 \mathrm{~m}$ en exposition sud s'étendent les Chênaies xérophiles méditerranéennes à base de Chênes Verts et de Chênes-Lièges. Repoussée en soulane, la forêt de Chêne-Liège ne subsiste qu'à l'état de lambeaux. Elle a cependant conservé l'essentiel de son originalité floristique et physionomique. La Subéraie, régulièrement démasclée, se présente comme une formation claire à sous-bois de xérophytes et d'héliophytes comme le Pistachier, le Lentisque, l'Arbousier, le Jasmin, la Bruyère arborescente, les Cistes à feuilles de sauge. Lorsque la forêt disparaît, il se forme un maquis à base d'Arbousiers et de Térébinthes. Sur les versants décapés ne subsiste plus qu'une formation ouverte à Labiées. Le Chêne Vert et son cortège paraissent plus solidement implantés. Les espèces sont moins exigeantes et l'on voit apparaître quelques plantes atlantiques dans les sous-bois les plus humides, en particulier le Genêt occidental (Genista hispanica occidentalis). Dans les formations dégradées, biologiquement plus sèches, les plantes méditerranéennes reprennent le dessus, avec le Filaire, l'Alaterne, le Prunier Mahaleb, le Chèvrefeuille d'Etrurie, etc. Le terme de l'évolution est représenté par une sorte de garrigue odorante à Lavande stoechas, Thym commun, Badasse, Thapsia velue. Quelques espèces atlantiques parviennent à s'infiltrer dans ces différentes formations; ce sont les héliophiles de la lande : Bruyères et Daboécie, plus rarement le Sarothamne cantabrique et l'Ajonc d'Europe. Dans l'ensemble, les formations méditerranéennes paraissent se régénérer sur place et jouir d'un relatif équilibre malgré les dégâts causés par les défrichements et la vie pastorale.

Les conditions écologiques sont, il est vrai, exceptionnellement favorables. La Liébana est un îlot de sécheresse bien protégé, au Nord, par le Massif des Picos de Europa. Au centre de la cuvette. les précipitations tombent au-dessous de $600 \mathrm{~mm}$ avec deux mois de sécheresse ( $\mathrm{Pmm}<2 \mathrm{~T}^{\circ}$ en juillet et août). De plus, l'atmosphère est asséchée par un effet de foehn sur le versant sud des Picos (2).

B) Gorges et bassins abrités des Picos de Europa

Au sortir de la Liébana, le Deva s'enfonce dans le Massif oriental des Picos. La « caliza de Montaña »du Dinantien, calcaire cristallin profondément karstifié, donne des gorges aux parois subver-

(2) Etude plus détalllée : G. Bertrand, n॰ 2 et G. Bretrand - G. Dupias, no 3 (cr. bibliographle in fine). 
ticales. Une végétation adaptée, calcicole et rupicole, pauvre en espèces, s'aligne dans les réseaux de diaclases. Les arbustes sempervirents dominent : Chêne Vert, Filaire, Laurier sauce, quelques Térébinthes. Ils forment une couverture discontinue qui grimpe le long des parois jusqu'à plus de $1.000 \mathrm{~m}$. Les gorges du Deva exercent donc une première sélection parmi les plantes avant de s'ouvrir sur le minuscule bassin de Lebeña.

Entre des parois calcaires, surchauffées en été, se creuse vers $250 \mathrm{~m}$ d'altitude une dépression d'une quinzaine de $\mathrm{km}^{2}$ correspondant à une petite pincée synclinale dans les schistes du Culm. Abrité de tous les côtés, le bassin de Lebeña constitue la station biogéographique la plus chaude et certainement la plus sèche de toute la région cantabrique. Par rapport à la Liébana, on note - en été et par beau temps stable - 1 à $2^{\circ} \mathrm{C}$ en plus et 5 à $10 \%$ d'humidité relative en moins. Les plantes atlantiques disparaissent presque totalement. Parmi les méditerranéennes triomphent les thermophiles et les héliophiles comme le Lentisque et le Jasmin jaune. Toutes ces espèces se mêlent dans les mêmes stations (3) :

Route de Panes à Potes $(230 \mathrm{~m}$ ) Bois d'Allende $(450 \mathrm{~m})$

(sur schistes décapés) (sur éboulis calcaire)

Arbres :

Chêne Vert : 1 Chêne Vert :

Filaire :

2 Chêne-Liège : 2

Arbousier (en arbre) : 1

Strate frutescente:

Jasmin jaune :

2

Térébinthe :

Strate sous-frutescente :

Chèvrefeuille d'Etrurie : 1

Salsepareille :

Cistes à feuilles de

Hellébore fétide : 1

Bruyère cendrée : $\quad+$

En aval de Lebeña le cortège du Chêne Vert se modifie lentement. Un premier « échelon d'appauvrissement 》 (Ch. Flahault, P. Rey) prend place entre Lebeña et la Hermida. Plusieurs espèces physionomiquement importantes disparaissent totalement. C'est le cas du Chêne-Liège et d'espèces xérophiles eu-méditerranéennes : le Jasmin, l'Oxycèdre, la Lavande stoechas, la Bruyère arborescente, le Lotier à feuilles de cytise, etc. Ces plantes sont relayées au sein des formations végétales par d'autres, moins exigeantes.

(3) Les plantes sont classées d'après la surface couverte (abondance-dominance), + : plantes rares, 1 : plantes abondantes mals ne recouvrant pas de surface appréciable, $2: 10$ à $25 \%, 3: 25$ a $50 \%, 4: 50$ a $75 \%, 5: 75$ a $100 \%$. 
Sans qu'il s'agisse d'une substitution terme à terme, on peut dire cependant que la Bruyère à balais remplace l'Arborescente, le Genévrier commun, l'Oxycèdre, le Filaire, l'Arbusier, etc. Enfin, le Laurier sauce et l'Epine-vinette prennent une notable expansion.

\section{C) Infiltrations atlantiques et montagnardes dans les basses gorges du Deva}

En aval de Lebeña quelques torrents issus des Picos de Europa viennent confluer avec le Deva. Ils coulent au fond de petites gorges orientées Est-Ouest. Sur les éboulis qui tapissent les ombrées se sont glissées de nombreuses plantes originaires des hauts plateaux karstifiés. Le long de l' « arroyo » de Cicera, au Nord de la Hermida, des spécimens de l'étage « montagnard »: Hêtre, Houx, Blechnum spicant, se mêlent au Chêne Vert et à son cortège. Cette remarquable inversion d'étage souligne l'importance des migrations de plantes depuis le Post-glaciaire. L'apparition de ces espèces hygrophiles, dans un biotope bien défini il est vrai, annonce cependant une évolution écologique qui prend toute son ampleur à partir de la Hermida.

Au droit de ce village, un grand accident tectonique tranche le calcaire cristallin. Sur un bloc effondré sont coincés des paquets de Permo-Trias ployés en synclinal. Les argiles rouges et les calcaires gréseux conservés à la faveur de cet accident sont colonisés par une lande subatlantique à base d'Ajonc d'Europe, de Sarothamne cantabrique, de Callune, d'Ericacées et par de belles Châtaigneraies mélangées à quelques bosquets de Rouvre. Les Chênes Verts qui subsistent en dehors des gorges du Deva, ou bien ont perdu leur sous-bois « méditerranéen 》 au profit du Genêt occidental et de la Bruyère à balais, ou bien s'accrochent, en inversion d'étage, sur les hautes soulanes calcaires qui dominent le synclinal, vers $700-900 \mathrm{~m}$ d'altitude. Ce changement floristique ne traduit pas seulement une modification dans la nature des sols qui sont en moyenne plus argileux et plus frais que dans les stations précédentes. Faute de données chiffrées, la simple observation montre que la région de la Hermida constitue une limite climatique entre un versant nord hyperhumide et une zone intra-montagnarde beaucoup moins arrosée. En effet, si les masses d'air humide formées sur le Golfe Cantabrique franchissent couramment le palier nord des Picos de Europa (1.000 $1.600 \mathrm{~m}$ ), elles viennent par contre se bloquer contre le massif méridional beaucoup plus élevé $(1.800-2.600 \mathrm{~m})$. Le contact entre ces deux compartiments tectoniques correspond au « graben » de la Hermida. La nébulosité devient subitement plus forte et le degré hygrométrique tombe rarement au-dessous de 65-75\%. La gorge du Deva en aval de la Hermida est, en effet, presque verdoyante. La lande à Fougère-aigle recouvre les vieux éboulis. Le Chêne Vert reste l'essence dominante, mais il perd peu à peu les éléments les plus caractéristiques de son cortège. Seuls 
subsistent l'Epine-vinette, le Filaire et le Laurier. Sur les hautes parois tourmentées par la karstification, le Chêne Vert pousse au milieu des Hêtres jusque vers $1.000 \mathrm{~m}$. Par contre, il laisse les stations humides du bord des eaux aux Tilleuls et aux Hêtres, ces derniers descendant à $60 \mathrm{~m}$ d'altitude. Dans les biotopes encore plus humides abondent les Fougères : le Blechnum, la Scolopendre, la Capillaire noire, l'Asplenium trichomanes, etc. On vient de franchir un autre " échelon d'appauvrissement ». Avec les Picos de Europa et leurs stations abritées sur « caliza de montaña » finit le véritable domaine des formations méditerranéennes. Ces dernières se retrouvent pourtant au Nord des Picos, mais leur physionomie est fortement altérée et leur dynamique parait modifiée. Cependant, moins que leur effacement progressif, c'est au contraire les conditions de leur maintien en pleine zone océanique qui doivent retenir l'attention.

\section{LES STATIONS OCEANIQUES}

Les Picos de Europa sont brutalement interrompus par un grand fossé d'effondrement Est-Ouest drainé par le Cares qui vient confluer avec le Deva dans le bassin de Panes. Dans ce dernier, se sont conservés des calcaires gréseux datés du Cénomanien et du Nummulitique. Ces terrains, violemment plissés, viennent s'écraser au Nord contre le môle de la Sierra de Cuera, vigoureux relief de 500 à $1.100 \mathrm{~m}$ d'altitude, formé par les calcaires du Dinantien et les quartzites du Dévonien-Ordovicien (fig. 2). Avec le bassin de Panes commence le paysage végétal classique du littoral asturien et « montañés》: prairies toujours vertes, champs de maïs gorgés d'eau, bosquets de Chênes Verts ou de Chênes à feuilles caduques (Pédonculés, Sessiles) et reboisements d'Eucalyptus. La végétation se répartit en trois grands domaines que les déforestations et les reboisements systématiques rendent difficiles à cerner. La « série 》 du Hêtre occupe la Sierra de Cuera, à partir de $300 \mathrm{~m}$ sur le versant nord et seulement de $1.000-1.100 \mathrm{~m}$ sur le versant sud ce qui souligne le rôle fondamental de l'exposition. Dans les zones basses non calcaires dominent les Chênes caducifoliés avec des plantes de sous-bois humide : Houx, Anémone Sylvie (Anercone nemorosa). Blechnum spicant, qui sont aussi des espèces caractéristiques de la Hêtraie mésophile. Sur les affleurements calcaires s'installent les Chênes Verts, surtout lorsqu'il s'agit de soulanes.

\section{A) La soulane d'Alevia}

Elle se situe au contact entre les calcaires nummulitiques ployés en anticlinaux et le calcaire paléozoïque (fig. 2). Entre 90 et $450 \mathrm{~m}$ d'altitude s'étend un versant bien abrité où les affleurements de calcaire nu constituent d'excellents biotopes pour les espèces médi- 


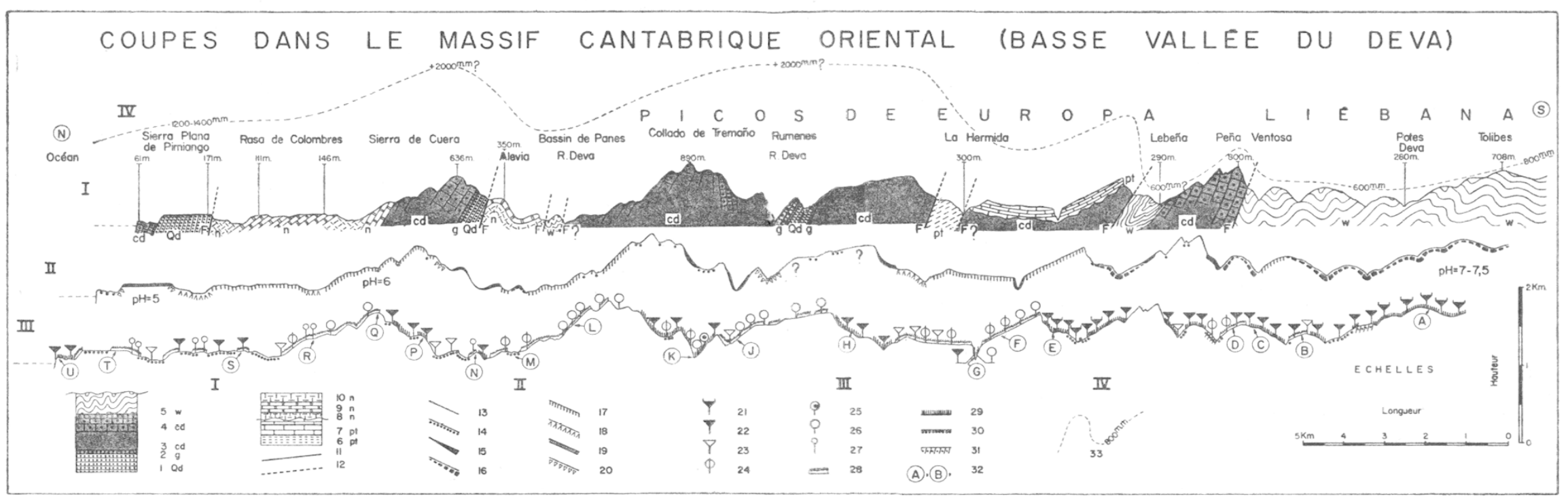

Figure 2. Coupes dans le massif cantabrique oriental (basse vallée du Deva).

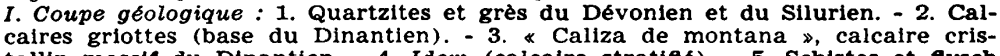
tallin massif du Dinantien. - 4. Idem (calcalre stratiffe). - 5. Schistes et flysch rouges du Permo-trias. - 8. Argiles et calcalres gréseux du Cénomanien. - 9-10. Calcalres et marnes du Nummulitique (?). - 11. Ligne de falle. - 12. Ligne de falle probable. - 11. Les grands types de 8ols 13 . Ameurements rocheux ou

terranéennes. La série du Chêne Vert domine entre 250 et $400 \mathrm{~m}$ d'altitude. Les bois ne subsistent qu'à l'état de lambeaux qu'envahissent les Eucalyptus. Dans le maquis, le nombre de plantes méditerranéennes est resté élevé : Filaire, Térébinthe, Alaterne, Arbousier, Amélanchier commun et quelques Chênes Verts buissonnants. Au stade de la « sous-fruticée », le caractère méditerranéen s'estompe. C'est le triomphe des rupicoles et des xérophiles de toutes origines : le Lilas d'Espagne (Centranthus ruber), l'Hellébore fétide, la Globulaire (G. nudicaulis), la Vipérine (Echium vulgare), le Fragon, le Panicaut de Bourgat, le Fenouil commun, etc. Ces espèces se mêlent aux Bruyères atlantiques et au Genêt occidental. A la base des versants, au milieu des vergers de pommiers et des châtaigneraies, on reconnait les traces d une ancienne Chênaie mixte à Chêne Vert et Pubescent. Vers le haut du versant, apparaît une lande à Ajoncs d'Europe. Callune et Daboécie qui assure une transition mal définie avec la Hêtraie. Les plantes méditerranéennes se disper-

Sols rendziniformes. - 16. Rankers A.C. de pente et sols calcimorphes de pente.
- 17. Sols bruns de pente. - 18. Sols argileux plus ou moins gleyifles. - 19. Sols 17. Sols bres a de perts la végétation. - 21. Chêne-Liège. - 22. Chene vert. - 23 . Chenes caducifollés. 28. Lande subatlantique et formations derivees. - 29. Maquis ou garrigue. Profl des précipitations (hypothétique).

sent donc dans les différentes formations atlantiques. En effet, les formations méditerranéennes ont "éclaté 》 et le Chêne Vert, repoussé sur les corniches calcaires, ne représente plus une tête de "série ». Ces faits pourraient laisser croire que la soulane d'Alevia constitue lultime "échelon d appauvrissement ». Aussi, ce n est pas sans surprise que l'on découvre les stations méditerranéennes des " rasas " et des falaises de la côte asturienne.

B) Les stations méditerranéennes du littoral

Au Nord de la Sierra de Cuera, s'étale une zone basse, de 50 à $250 \mathrm{~m}$ d'altitude, large de 2 à $3 \mathrm{~km}$ qui se termine en falaises sur l'Océan. Du point de vue biogéographique comme du point de vue géomorphologique, il faut distinguer les «Sierras Planas 》 des "Rasas ». Vers $200 \mathrm{~m}$ d'altitude, les «Sierras Planas 》 se présentent comme des tables d'une horizontalité parfaite, taillées 


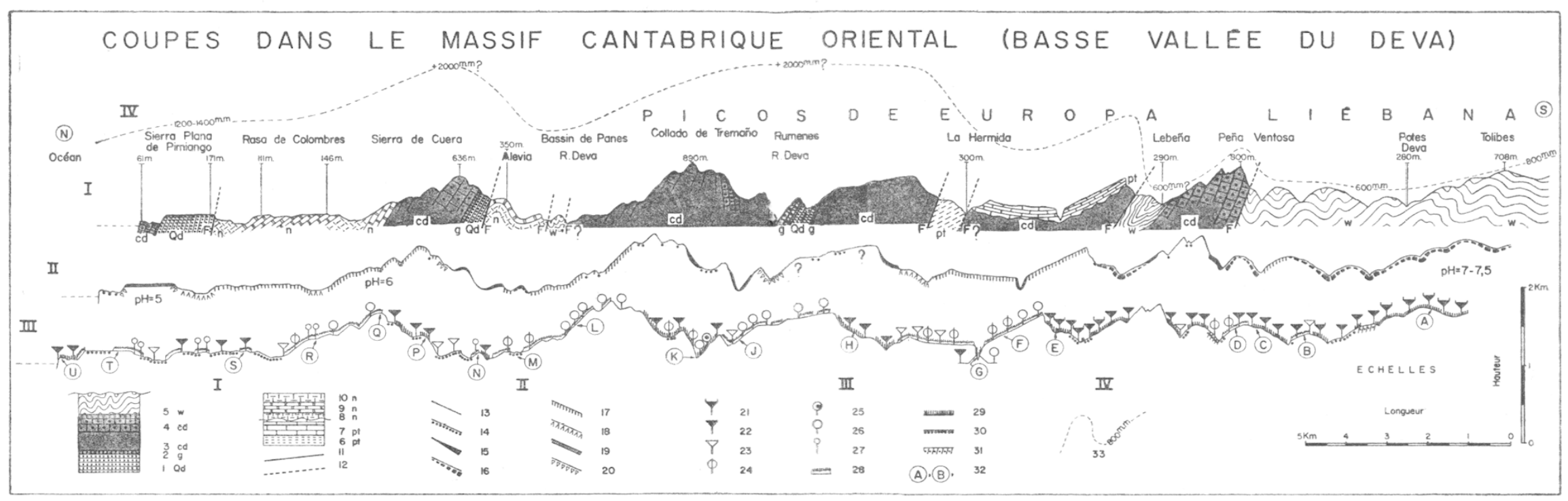

Figure 2. Coupes dans le massif cantabrique oriental (basse vallée du Deva).

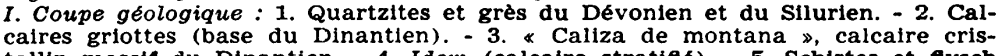
tallin massif du Dinantien. - 4. Idem (calcaire stratifle). - 5. Schistes et flysch rouges du Permo-trias. - 8. Arglies et calcalres gréseux du Cénomanien. - 9-10. Calcaires et marnes du Nummulitique (?). - 11. Ligne de fallle. - 12. Ligne de lalle probable. 11. Les grands

terranéennes. La série du Chêne Vert domine entre 250 et $400 \mathrm{~m}$ d'altitude. Les bois ne subsistent qu'à l'état de lambeaux qu'envahissent les Eucalyptus. Dans le maquis, le nombre de plantes méditerranéennes est resté élevé : Filaire, Térébinthe, Alaterne, Arbousier, Amélanchier commun et quelques Chênes Verts buissonnants. Au stade de la « sous-fruticée », le caractère méditerranéen s'estompe. C'est le triomphe des rupicoles et des xérophiles de toutes origines : le Lilas d'Espagne (Centranthus ruber), l'Hellébore fétide, la Globulaire (G. nudicaulis), la Vipérine (Echium vulgare), le Fragon, le Panicaut de Bourgat, le Fenouil commun, etc. Ces espèces se mêlent aux Bruyères atlantiques et au Genêt occidental. A la base des versants, au milieu des vergers de pommiers et des châtaigneraies, on reconnaît les traces d une ancienne Chênaie mixte à Chêne Vert et Pubescent. Vers le haut du versant, apparaît une lande à Ajoncs d'Europe. Callune et Daboécie qui assure une transition mal définie avec la Hêtraie. Les plantes méditerranéennes se disper-

Sols rendziniformes. - 16. Rankers A.C. de pente et sols calcimorphes de pente
17. Sols bruns de pente. - 18. Sols argileux plus ou moins gleyifies. - 19. Sols 17. Sols bruns de pente. 18. Sols argileux plus ou molns gley III Coupe do la végétation. - 21. Chêne-Liege. -22 . Chene Vert. - 23 . Chenes caducifollés. 24. Chataignier. -25 . Tilleul. - 26. Hêtre. - 27. Eucalyptus (reboisement).
28. Lande subatiantique et formations dérivees. - 29. Maquis ou garrigue. Profll des précipitations (hypothétique).

sent donc dans les différentes formations atlantiques. En effet, les formations méditerranéennes ont "éclaté 》 et le Chêne Vert, repoussé sur les corniches calcaires, ne représente plus une tête de "série ». Ces faits pourraient laisser croire que la soulane d'Alevia constitue lultime "échelon d appauvrissement ». Aussi, ce n est pas sans surprise que l'on découvre les stations méditerranéennes des " rasas » et des falaises de la côte asturienne.

\section{B) Les stations méditerranéennes du littoral}

Au Nord de la Sierra de Cuera, s'étale une zone basse, de 50 à $250 \mathrm{~m}$ d'altitude, large de 2 à $3 \mathrm{~km}$ qui se termine en falaises sur l'Océan. Du point de vue biogéographique comme du point de vue géomorphologique, il faut distinguer les «Sierras Planas 》 de "Rasas ». Vers $200 \mathrm{~m}$ d'altitude, les «Sierras Planas 》se présentent comme des tables d'une horizontalité parfaite, taillées 
dans les quartzites et les grès du Dévonien et du Silurien. Les plantes méditerranéennes n'arrivent pas à s'accrocher sur ces surfaces battues par les vents marins, où les sols sont à la fois hydromorphes et acides ( $\mathrm{pH}=4,5$ à 5,5). C'est le domaine de la lande rase à Ericacées (E. Tetralix, E. ciliaris) et à Ajoncs (Ulex nanus et U. Gallii) et des tourbières à Sphaigne, Polytricum, Drosera, etc. Dans les fonds de ravins ruisselants d'humidité se développent les Osmondes royales. En contre-bas, les « rasas 》 off rent des conditions écologiques très différentes bien que les précipitations soient, ici aussi, de l'ordre de 1.200 à $1.400 \mathrm{~mm}$. Ce sont des surfaces élaborées dans un matériel calcaire varié, paléozoïque ou tertiaire, formant un "karst-passoire 》 très accidenté. Chaque doline constitue une unité biogéographique bien circonscrite, pourvue d'un microclimat d'abri et de nombreux biotopes «secs 》 (lapiés dénudés). Les plantes et les formations méditerranéennes colonisent la totalité des " rasas 》 y compris les falaises et les petits îlots. Il s'agit, bien sûr, de formations très dégradées et, à part quelques bosquets de Chêne Vert à sous-bois mixte de Laurier sauce, Fragon, Salsepareille et Anémone hépatique (Hepatica triloba), on trouve surtout une sorte de «maquis-lande » impénétrable de 1 à $3 \mathrm{~m}$ de haut dont la composition floristique semble, au premier abord, assez étonnante (3).

«Rasa » de San Emeterio

(Grotte préhistorique du pindal, altitude $45 \mathrm{~m}$, à $100 \mathrm{~m}$ de la mer)

Strate frutescente :

Chêne Vert (en arbre et buissonnant) : 3

Ajonc d'Europe :

2

Arbousier :

Filaire :

Genêt occidental :

2

2

1

Strate sous-frutescente :

Salsepareille :

Bruyère vagabonde :

Cistes à feuilles de sauge : 2

Grémil (Lithospermum diff usum) :

etc.

\section{«Rasa » de Pechon}

(altitude $65 \mathrm{~m}$, même situation)

Ajonc d'Europe : 3

Chêne vert buissonnant : 2

Arbousier :

Laurier sauce :

2

Bruyère de Lusitanie (E. sitanica Rudolphi) : 1

Ciste à feuilles de Fragon : $\quad \begin{array}{ll} & \\ & \end{array}$

Daboécie :

2

Dans tous ces relevés, on note d'assez nombreuses plantes atlantiques; mais ces dernières ne paraissent jouer qu'un rôle subor-

(3) Cf. note 3 page 45 . 
donné et ne semblent jamais très envahissantes. L'étude de ces formations méditerranéennes en milieu hyperocéanique entraîne deux remarques : d'une part, les plantes calcicoles et silicicoles se retrouvent sur les mêmes biotopes ; d'autre part, les espèces méditerranéennes qui prospèrent dans la zone littorale sont presque uniquement des plantes laurifoliées.

Tout au long du littoral cantabrique, du Pays Basque francoespagnol à la Galice, on retrouve des formations analogues à celles de la vallée du Deva. Cette expansion des plantes méditerranéennes pose un problème d'ordre écologique qu'il faut, maintenant, examiner dans son ensemble.

\section{L'ADAPTATION ECOLOGIQUE}

Ces formations sont suffisamment étendues et suffisamment structurées pour qu'on ne puisse les regarder comme un simple phénomène aberrant sans signification sur le plan de la biogéographie régionale. Les explications relatives à leur mise en place et à leur conservation doivent être recherchées d'une part, dans les conditions écologiques offertes par la région nord cantabrique; d'autre part. dans les caractères propres aux espèces végétales en présence.

\section{A) $L^{\prime} \ll$ amplitude écologique »du versant nord cantabrique}

Les formations végétales méditerranéennes ne constituent pas les éléments avancés d'une frange pionnière. Réfugiées dans les stations abritées, coupées les unes des autres et uniquement composées de plantes à aires fragmentées, elles ont, au contraire, tous les caractères de « relictes » en déséquilibre avec les conditions bioclimatiques. Leur mise en place, inconcevable de nos jours, a dû s'effectuer au moment de la période dite xérothermique. Il est difficile de préciser davantage car les rares analyses polliniques effectuées dans la région ne fournissent aucun renseignement sur ces migrations (4). Entre le monde méditerranéen et la frange cantabrique à climat océanique s'interpose la double barrière des «paramós» de Vieille Castille, glacés en hiver, et du Massif cantabrique dont les rares cols sont à plus de $1.400 \mathrm{~m}$ d'altitude. L'absence de formations méditerranéennes bien caractérisées sur le versant sud de ce massif, il est vrai plus élevé et plus continental, laisse supposer que les infiltrations se sont effectuées d'Est en Ouest, le long du littoral, à partir des provinces basques espagnoles où les conditions de passage paraissent meilleures (altitudes moins fortes, larges affleurements de calcaire, proximité du bassin de l'Ebre). Cependant, il

(4) MENENdez Amor J., Y Florschutz F. : Sur les elements steppiques dans la végétation quaternaire de l'Espagne. Bol. Real. Soc. Esp. de Hist. Natural, 1963, no 61, pp. 121-123, graphiques. Et MENENDEZ AMOR J., Y FlorschuTz F. : Contribucion al conocimiento de la historia de la vegetacion en Espana durante el Cuaternario. Est. geologicos, 1961, vol. XVII, no 1, pp. 3-19, graphiques. 
n'est pas exclu que certaines méditerranéennes à caractère steppique aient progressé sur les plateaux castillans. C'est, par exemple, le seul moyen d'expliquer les stations de Genévrier-Thurifère de la haute vallée de l'Esla. De toute manière, il ne semble pas y avoir eu beaucoup de contacts floristiques entre les deux versants. Les stations méditerranéennes sont l'un des traits originaux du versant nord.

Pourtant, les caractères écologiques de ce versant paraissent en moyenne, assez éloignés de ceux des régions méditerranéennes. La surabondance des précipitations et les valeurs élevées du degré hygrométrique constituent, en apparence du moins, des obstacles majeurs. Cependant, une analyse plus fine laisse entrevoir un certain nombre d'analogies avec le climat méditerranéen. L'été est la saison la moins arrosée et une véritable sécheresse biologique apparaît dans les stations abritées. C'est, on l'a déjà noté, le cas pour la Liébana ( $\mathrm{Pmm}<2 \mathrm{~T}^{\circ}$ pour juillet et août). Les saisons intermédiaires totalisent les grosses chutes d'eau. Le maximum se place en automne, c'est-à-dire à une époque où l'eau est peu utile aux plantes. Le rythme annuel des précipitations étant le même que dans la région méditerranéenne, le cycle végétatif peut donc se dérouler normalement. L'adaptation des plantes n'est donc pas d'ordre qualitatif, mais simplement quantitatif. De toute manière, l'excès d'eau est localement contrebalancé par une série de « correctifs écologiques ». Il y a, d'abord, le calcaire qui affleure largement dans toute la région. Il donne un milieu sec sur lequel se réfugient la plupart des méditerranéennes, les silicicoles aussi bien que les calcicoles. Les pentes, vigoureuses dans toute la région, augmentent encore la sécheresse biologique en favorisant le ruissellement aux dépens de l'infiltration et en bloquant la pédogénèse. En effet, la minceur et l'instabilité mécanique des sols constituent un fait presque général qu'il s'agisse de Rankers, de sols rendziniformes ou de sols bruns lessivés obliquement. En définitive, on peut admettre que les 1.200 à $1.500 \mathrm{~mm}$ de précipitations annuelles ne sont plus de rigoureux « facteurs-limitants »quand ils s'appliquent à des stations où interviennent, souvent en même temps, ces « correctifs écologiques », ce qui est un cas assez fréquent sur les soulanes du Massif Cantabrique.

Les températures ne posent pas de problèmes d'adaptation particuliers. Le climat est de type océanique chaud (moyenne annuelle $13-14^{\circ}$, moyenne d'août : $\left.18^{\circ}\right)$. Mais il y a un fait encore plus important. L'infiltration des plantes méditerranéennes s'explique en grande partie parce que le plus rigoureux des «facteurs-limitants ». le cycle gel-dégel, est peu fréquent dans une région où les oscillations thermiques sont amorties par la masse océanique (moyenne de janvier : $\left.9^{\circ}\right)$. Or, il semble que ce soit le gel qui détermine la limite septentrionale et occidentale de nombreuses espèces méditerranéennes dans les régions continentales des plaines de l'Ebre ef des « paramós » de Castille, car les plantes à feuilles persistantes 
sont désarmées contre les fortes gelées hivernales. Le versant nord cantabrique possède donc une «fourchette écologique » suffisamment ouverte pour que certaines plantes subméditerranéennes, ou même quelques eu-méditerranéennes, installées pendant la période dite xérothermique puissent se maintenir dans les stations privilé giées surtout si l'on tient compte de la remarquable plasticité de la plupart d'entre elles.

\section{B) Plasticité et concurrence des espèces}

Les conditions écologiques, simples virtualités, n'expliquent pas toujours de façon satisfaisante la localisation. la structure ou même la dynamique de certaines formations méditerranéennes. La concurrence entre les espèces, leur plus ou moins grande plasticité, jouent un rôle certainement important mais malaisé à définir. Tout au plus, peut-on formuler quelques hypothèses.

Le premier problème, déjà posé par P. Allorge $\left(n^{\circ} 1\right)$ à propos du Pays Basque, a trait aux espèces laurifoliées, c'est-à-dire au Chêne Vert, au Chêne-Liège, à l'Arbousier, au Laurier sauce, au Filaire, etc. On a déjà remarqué que ces plantes représentaient le dernier « échelon d'appauvrissement » et qu'elles se maintenaient parfaitement bien sur le littoral. On les retrouve dans toute la région méditerranéenne humide et, assez fréquemment, sur la façade atlantique tiède de l'Europe jusqu'en Irlande et en Norvège (5). $\mathrm{Ne}$ peut-on pas y voir des relictes d'une flore tertiaire chaude et humide de type «pontique », qui aurait pu, par la suite, se mélanger à la flore méditerranéenne, mais qui serait plus ou moins autochtone dans la région cantabrique? Ainsi, on comprendrait mieux pourquoi ces plantes relaient les véritables méditerranéennes sur le littoral et aussi pourquoi elles sont, dans l'ensemble, beaucoup plus envahissantes par rapport aux formations atlantiques. Dans cette hypothèse, leur signification écologique demanderait à être révisée. Le qualificatif de « méditerranéen 》 accolé à ces plantes n'est-il pas un abus malgré l'évidence de certains critères chorologiques? Le Chêne Vert en est, en quelque sorte, le symbole ; il mériterait une étude écologique spéciale. On peut simplement souligner son étonnante plasticité à travers toute la région cantabrique. Indifférent au $\mathrm{pH}$ du sol, accroché aux îlots du littoral ou aux parois des Picos de Europa, il s'associe aussi bien au Hêtre qu'au Lentisque. Mais n'y aurait-il pas des variétés différentes? Si l'on veut conserver la « série » du Chêne Vert il faut distinguer trois faciès : un « faciès xérophile » correspondant aux stations intramontagnardes d'abri avec d'indiscutables espèces méditerranéennes, un « faciès mésophile 》 dans lequel s'effectue le passage aux espèces laurifoliées mais où subsistent encore de nombreuses subméditerra-

(5) Voir en particulier J. K. Charlesworth, G. F. Mitchell, J. Heslop haraIson, etc. : The History of the Irish flora and fauna. Belfast Meeting of the British Association (feulllets polycopiés), 9 septembre 1952. 
néennes, enfin, un « faciès hygrophile » à base d'espèces « laurifoliées » (fig. 1). Un autre aspect, très géographique, de la plasticité et de la concurrence entre les espèces, est le mélange des calcicoles et des silicicoles non seulement dans les mêmes stations mais encore sur les mêmes biotopes (diaclase par exemple). Presque toutes les méditerranéennes, y compris le Chêne-Liège et l'Arbousier, poussent sur le calcaire nu, sur des sols rendziniformes ou calcimorphes. A la limite de leurs aires respectives ces plantes se réfugient dans les stations chaudes et sèches, les seules qu'elles puissent disputer aux plantes atlantiques. La concurrence entre les formations méditerranéennes et atlantiques est surtout intéressante au niveau du maquis et la lande. Le maquis, ou la garrigue, gagnent sur la lande à la périphérie des grandes stations méditerranéennes, au contraire, ils perdent du terrain quand les stations méditerranéennes sont peu étendues et mal équilibrées. Cet « effet de masse » est en train de menacer sérieusement toutes les petites stations méditerranéennes. A l'intérieur même des Chênaies méditerranéennes, la « série » du Chêne-Liège est ainsi en train de se fragmenter, sinon de disparaître au profit du Chêne Vert. Les plantes et les formations végétales méditerranéennes du versant nord cantabrique «symbolisent, pour reprendre une expression de $M$. Pierre Birot, ce qu'il y a d'équivoque dans ce type de climat » (6) du Nord-Ouest de la Péninsule Ibérique qui participe à la fois du régime atlantique et du régime méditerranéen. On peut parler d'une région biogéographique « lusoméditerranéenne ». Le « déterminisme écologique », particulièrement élastique dans ce cas, favorise la diversification du tapis végétal. Cependant, il n'est pas le seul responsable de la structure de la végétation. La concurrence pour l'espace que se livrent des plantes et des formations particulièrement «plastiques » constitue aussi une des bases de l'explication des paysages végétaux.

\section{BIBLIOGRAPHIE SOMMAIRE}

1. Allorge Plerre : Essai de synthèse phytogéographlque du pays basque. Bull. Soc. Botan. France, 1941, tome LXXXVIII ext. Pays basque, pp. 291-356, Paris.

2. Bertrand Georges : Esquisse blogéographique de la Liébana (massif cantabrique, Espagne) : la dynamique actuelle des paysages. Rev. geogr. Pyr. et S.O., 1964, no 3, 2 pl. phot., 7 cartes, 1 carte h.t. couleur au 1/100.000.

3. BERTRAND Georges et DUPias Georges : Contribution à l'étude phytogéographlque des réglons cantabriques (Espagne du Nord-Ouest) : I. Les vallees de Líbbana. Bull. Soc. Hist. Nat., Toulouse (sous presse).

(6) Plerre Birot : Le Portugal, 1950, Paris, Armand Colín, p. 30. 
4. BIRot Pierre : La Méditerranée et le Moyen-Orient. Tome I : La Méditerranée occidentale, P.U.F., Paris, 1953, pp. 43-88.

5. DuPoNr Pierre : La flore atlantique européenne. Introduction à l'étude du secteur ibéro-atlantique. Thèse Sciences, Toulouse. Documents pour les cartes des productions végétales. Série : Europe-Atlantíque, 1962, 1 vol., 414 p.

6. GuINEA LOPEZ Emllio : Geografla botanica de Santander. Diputacion provincial de Santander. Santander, 1953, 295 p.

7. LASCombes Georges : La végétation des Picos de Europa. Les paysages lorestlers. Bull. Soc. Hist. Nat., Toulouse, i944, tome LXXIX, 40 trim., pp. 339-359. 1 carte au $1 / 100.000$.

\section{DISCUSSION}

M. Dresch : 1. Quelles sont les relations entre les reliques méditerranéennes, le Chêne-Liège en particulier et les sols actuels et anciens?

2. Quelles sont les données de la palynologie?

M. Bertrand : Le Chêne-Liège est accompagné d'un cortège d'espèces xérophiles dont certaines sont $d$ 'indiscutables méditerranéennes comme Lavandula stoechas, Jasminum fruticans, Pistacia Lentiscus, P. Térébinthus, etc. Il s'agit donc d'une véritable \& formation $\gg$ bien structurée et non de plantes méditerranéennes éparpillées dans des séries atlantiques.

- Les sols sont en général des sols bruns forestiers plus ou moins dégradés et des rankers de pente. Il n'a pas été trouvé de paléosols rouges. Dans l'ensemble, les sols sont très dégradés, parfois entièrement décapés (effet combiné des fortes pentes et de la surpécoration). Le sol « climacique » de la Subéraie à Arbousier est un sol brun forestier.

- Les analyses palynologiques réalisées dans la région cantabrique intéressent surtout les plantes « humides 》 et acidiphiles. Elles n'apportent rien de positif pour la connaissance des migrations de plantes méditerranéennes.

M. GEORGE : Dans le cas d'une destruction totale de la végétation en une station mixte donnée, y a-t-il différenciation dans la reconstitution?

M. Bertrand : Sur un versant nord dans la série du Chêne Vert (vers $850 \mathrm{~m}$, près de Potes, Liébana) les forestiers ont dégagé des laies forestières dans un maquis à Filaire et Arbousier. Sur les sols minces, les plus fréquents, apparaissent d'abord les Cistes à 
feuilles de sauge, puis des plantules d'Arbousiers. Le maquis se reconstitue par la suite, avec une physionomie comparable au faciès antérieur. Par contre, sur les sols plus profonds et plus humifères s'installe la Fougère-aigle et les Châtaigniers.

M. Guilcher : A côté des contrastes Sud-Nord (raréfaction des plantes méditerranéennes dans cette direction), l'auteur a-t-il observé des contrastes Est-Ouest, que d'autres ont noté dans la végétation d'algues littorales?

M. Bertrand : La dégradation Sud-Nord de la végétation méditerranéenne se retrouve aussi dans le sens Est-Ouest tout au long du littoral cantabrique. Elle est liée au renfoncement des conditions atlantiques à mesure que l'on se rapproche de la Galice. Elle doit aussi être mise en rapport avec la dynamique migratoire des formations méditerranéennes qui ont dû coloniser le versant nord cantabrique à partir du bassin de l'Ebre et des provinces basques. Si certaines plantes méditerranéennes atteignent le littoral galicien les «formations méditerranéennes 》 ne semblent pas dépasser l'Est asturien. 